\title{
Respuestas autonómicas reducidas ante estímulos emocionales en el alcoholismo: la relevancia de la impulsividad
}

\author{
Blunted autonomic responses to emotional stimuli \\ in alcoholism: relevance of impulsivity
}

\author{
Martina Carmona-Perera***, Xavier Sumarroca-Hernández***, Angelina Santolaria- \\ Rossell***, Miguel Pérez-García***********, Gustavo A. Reyes del Paso******. \\ * Departamento de Personalidad, Evaluación y Tratamiento Psicológico. Facultad de Psicología, Universidad de Granada, \\ Campus Cartuja s/n. 18071 Granada, España. ** Fundació Villablanca, Serveis Assistencials. Hospital Universitari Institut Pere \\ Mata, Ctra. Bellissens s/n. 43204 Reus, España. *** Unidad de Conductas Adictivas (UCA). Hospital Nostra Senyora de \\ Meritxell, C/ dels Escalls, s/n. AD700 Escaldes-Engordany, Andorra. **** Centro de Investigación Mente, Cerebro y \\ Comportamiento (CIMCYC). Universidad de Granada, Campus Cartuja s/n. 18071 Granada, España. ***** Centro de \\ Investigación Biomédica en Red de Salud Mental (CIBERSAM). Universidad de Granada, Av de Madrid nº 11, 18071 Granada, \\ España. ****** Departamento de Psicología. Universidad de Jaén, Campus de las Lagunillas s/n. 23071 Jaén, España.
}

\section{Resumen}

La dependencia al alcohol está asociada con anormalidades en el procesamiento de las emociones y comportamientos impulsivos en la búsqueda de alcohol. Sin embargo, pocos estudios han analizado las respuestas emocionales hacia estímulos motivacionalmente relevantes en personas dependientes al alcohol usando medidas tanto autonómicas como subjetivas. En este estudio se analizó la tasa cardiaca (TC), la conductancia de la piel (CP) y las respuestas subjetivas a estímulos emocionales cotidianos en individuos dependientes al alcohol, y su asociación con la impulsividad y el consumo de alcohol. La TC y la CP fueron registradas en 28 participantes dependientes al alcohol y en 31 participantes sanos durante la visualización pasiva de imágenes emocionales placenteras, desagradables y neutras. Posteriormente, los participantes evaluaron valencia, activación y dominancia de las imágenes y completaron la Escala de Impulsividad de Barratt. Los participantes dependientes mostraron respuestas reducidas en TC y CP, tanto hacia las imágenes emocionales positivas como negativas. En el caso de la CP, estas respuestas se asociaron a la impulsividad. Los participantes dependientes al alcohol mostraron una disociación entre las respuestas fisiológicas y subjetivas, con unas respuestas autonómicas disminuidas y unas respuestas subjetivas normales. Estos resultados sugieren que los individuos dependientes al alcohol tienen problemas en utilizar el feedback fisiológico emocional al responder a estímulos emocionales relevantes, y proporcionan una evidencia inicial de la contribución de la impulsividad a los déficits de procesamiento emocional en esta población. Estos resultados son congruentes con el papel clave que juega la experiencia emocional y la impulsividad en el abuso de sustancias. Palabras clave: Dependencia al alcohol; Respuestas autonómicas; Rxperiencia emocional; Impulsividad; Tasa cardíaca; Conductancia de la piel.

\begin{abstract}
Alcohol dependence is associated with abnormalities in the processing of emotional signals and impulsive alcohol-seeking behaviours, and these alterations compromise the effectiveness of treatment approaches. However, there is a lack of studies linking the experience of emotions to everyday motivationally relevant stimuli in alcohol-dependent individuals using both autonomic and selfreport measures. We analysed heart rate (HR), skin conductance (SC) and subjective emotional reactivity to everyday affective stimuli in alcohol-dependent individuals, and their associations with impulsivity and degree of alcohol consumption. SC and HR were continuously monitored in 28 alcohol-dependent individuals and in 31 non-alcohol healthy controls during passive viewing of pleasant, unpleasant, and neutral emotional pictures. Participants assessed the pictures for valence, arousal, and dominance and completed the Barratt Impulsiveness Scale. Alcohol-dependent individuals showed reduced $\mathrm{HR}$ and $\mathrm{SC}$ reactivity to both positive and negative emotional stimuli. In the case of SC, this blunted response was associated with impulsivity. Furthermore, alcohol-dependents displayed decoupled physiological and subjective responses, with blunted autonomic responses and normal subjective reports regarding emotional stimuli. Our findings indicate that alcohol-dependent individuals failed to use emotional autonomic feedback in response to natural, emotionally relevant stimuli, and provide initial evidence of the contribution of impulsivity to emotional processing deficits in this population. These results are in keeping with the proposed key role played by emotional experience and impulsivity in substance abuse.

Keywords: Alcohol-dependent individuals; Autonomic responses; Emotional experience; Impulsivity; Heart rate; Skin conductance.
\end{abstract}

Recibido: Noviembre 2017; Aceptado: Diciembre 2017.

Enviar correspondencia a:

Gustavo A. Reyes del Paso. Departamento de Psicología. Universidad de Jaén. 23071 Jaén, España. Fax: +34953211881.

E-mail: greyes@ujaen.es. 
$\mathbf{L}$ a dependencia al alcohol se relaciona con anormalidades en el procesamiento de señales emocionales, que pueden subyacer deficiencias en la toma de decisiones, los trastornos de ánimo, y la baja calidad de las interacciones sociales (Handelsmanet et al., 2000; Kornreich et al., 2002; Verdejo-García y Bechara, 2009), por tanto fomentando la recaída después de la abstinencia (Allsop, Saunders y Phillips, 2000; Bechara y Damasio, 2002). Por ejemplo, algunos estudios han mostrado la implicación de la mala regulación emocional tanto como de la mala expresión emocional en el alcoholismo (Foisy et al., 2007; Uekermann y Daum, 2008), y habían anteriormente asociado dichos déficits con las deficiencias sociales observadas en esta población (Kornreich et al., 2002; Maurage, Campanella, Philippot, Martin y Timary, 2008). Los estudios también han mostrado déficits en la toma de decisiones, parcialmente debido a déficits en el reconocimiento y la regulación de señales emocionales relacionados con las consecuencias negativas de decisiones potenciales, incluyendo la toma de decisiones de riesgo (Fernández-Serrano, Pérez-García, Schmidt Río-Valle y Verdejo-García, 2010; Park et al., 2010), y la toma de decisiones en el ámbito social (Breversa et al., 2013; Carmona-Perera, Reyes del Paso, Pérez-García y Verdejo-García, 2013; Carmona-Perera, Clark, Young, Pérez-García y Verdejo-García, 2014).

Además, los pacientes con dependencia al alcohol mostraron un amplio espectro de trastornos emocionales y de ánimo, incluyendo depresión, apatía, afecto aplanado, ansiedad y problemas en controlar la impulsividad (Bjork, Hommer, Grant y Danube, 2004; Mitchell, Fields, D'Esposito y Boettiger, 2005; Moselhy, Georgiou y Kahn, 2001; Stephens y Duka, 2008). Modelos neuropsicológicos recientes han destacado la contribución de la impulsividad en el control de respuestas significativas motivadas por la recompensa (Bechara, 2005; Carou, Romero y Luengo, 2017; Goldstein y Volkow, 2002; Verdejo-García, Lawrence y Clark, 2008). Por tanto, la disfunción emocional puede tener una asociación con problemas de control de los impulsos en respuesta a estados emocionales significativos (Carou et al., 2017; Kreusch, Vilenne y Quertemont, 2013; Verdejo-García, Rivas-Pérez, Vilar-López y Pérez-García, 2007). Estudios con neuroimágenes muestran de forma consistente que el uso crónico del alcohol produce ciertos efectos tóxicos en las áreas del lóbulo frontal involucradas en el procesamiento de las emociones y en el autocontrol conductual (Beck et al., 2012; Stephens y Duka, 2008).

En línea con la desregulación de los mecanismos de refuerzo (Robinson y Berridge, 2001, 2003), el componente emocional de las enfermedades adictivas se caracteriza por el realce del valor emocional de los refuerzos adictivos y la elevación de los umbrales de los refuerzos naturales. De esta manera, puede ser difícil para las personas con adic- ción sustituir sus conductas adictivas por otras actividades que conlleven refuerzos por su naturaleza. La hipótesis de los marcadores somáticos en la adicción (Verdejo-García y Bechara, 2009) también propone que aberraciones en la experiencia de emociones negativas explica los déficits en la toma de decisiones. La atenuación o la ausencia de respuestas autonómicas (marcadores somáticos) también puede explicar la reducción de la capacidad para considerar los estímulos relevantes naturales y emocionalmente significativos (Ferguson y Katkin, 1996; Goldstein y Volkow, 2002; Roedema y Simons, 1999).

Aunque aún no hay evidencia de este fenómeno en personas con adicción al alcohol, estos planteamientos han recibido apoyo por estudios comportamentales y psicofisiológicos con personas adictas a los opioides (Aguilar de Arcos et al., 2008; Chicharro, Pérez-García y Sanjuán 2011; Gerra et al., 2003; Lubman et al., 2009). En parte, dichos estudios aportan apoyo de las respuestas anormales subjetivas y neuroendocrinológicas a estímulos que ocurren de manera natural. Estudios psicofisiológicos con personas adictas a los opioides también han sugerido la desregulación de las respuestas emocionales a estímulos emocionales naturales. Por ejemplo, Gerra et al. (2003) informaron de tasas cardiacas (TC) disminuidas en respuesta a estímulos desagradables en esta población, y Lubman et al. (2009) hallaron respuestas disminuidas a estímulos agradables en electromiografías. De forma similar, también se ha hallado una conductancia de la piel (CP) reducida ante los refuerzos en personas con adicción al juego (Lole, Gonsalvez, Blaszczynski y Clarke 2012). No obstante, ninguno de estos estudios examinó la capacidad de respuesta emocional y psicofisiológica en personas con dependencia al alcohol.

En su conjunto, la evidencia anterior sugiere déficits en el procesamiento de las emociones por parte de personas con dependencia al alcohol, que pueden estar asociadas con la conducta impulsiva de búsqueda de alcohol de esta población. No obstante, sus reacciones emocionales ante estímulos cotidianos (no relacionados con las sustancias) relevantes en términos motivacionales, y el papel que asume la impulsividad en dichas reacciones, son aún en gran parte desconocidos. Este estudio tuvo como objetivo analizar las respuestas subjetivas (valencia, activación y dominancia) y psicofisiológicas (TC y CP) ante imágenes agradables, desagradables y neutras en personas con dependencia al alcohol abstinentes (DA) y en los participantes del grupo de control sanos (CS). La deceleración de la TC ha sido interpretada como indicador de vigilancia y control de la atención, tanto en la tradición occidental (e.g., Barry, 2006; Lacey y Lacey, 1970; Tremayne y Barry, 2001) como en la rusa (i.e., reflejo de orientación; Sokolov, 1963). En los paradigmas de visión pasiva, la TC también se ha considerado como indicador de la dimensión de valencia de las emociones (Lang, 
1995), en la cual la TC normalmente disminuye tras ver imágenes con valencia negativa y alto valor motivacional (Lang, Greenwald, Bradley y Hamm, 1993; Palomba, Sarlo, Angrilli, Mini y Stegagno, 2000). Tradicionalmente, la CP se ha usado como un índice fisiológico de activación (Lang, 1995), dado que la CP tiende a aumentar durante emociones excitantes y a disminuir durante emociones relajantes (Barry et al., 2004). La impulsividad se ha asociado con trastornos emocionales significativos en usuarios de drogas (Verdejo-García et al., 2007). En este contexto, también evaluamos la relación entre niveles de impulsividad, consumo de alcohol, y capacidad de respuesta emocional.

Nuestra hipótesis era que las personas con dependencia al alcohol mostrarían respuestas emocionales deficientes a estímulos naturales y emocionalmente significativos, en comparación con las personas del grupo de control sanas. Teniendo en cuenta las hipótesis de desregulación de refuerzos y de marcadores somáticos (Robinson y Berridge, 2003; Verdejo-García y Bechara, 2009), estos déficits deberían darse ante ambos estímulos placenteros y desagradables. De forma concreta, predijimos lo siguiente en los participantes con dependencia al alcohol: (i) respuestas subjetivas más bajas, tanto en las valoraciones de valencia como de activación; (ii) reactividad de la TC y la CP disminuidas, reflejado en una deceleración pronunciada de la TC y respuestas más bajas de la CP ante estímulos emocionalmente significativos positivos y negativos; y (iii) una asociación negativa entre puntuaciones en impulsividad y respuesta fisiológica, y una asociación positiva entre puntuaciones en impulsividad y consumo de alcohol.

\section{Métodos}

\section{Participantes}

La muestra estaba compuesta de 28 personas caucásicas con dependencia al alcohol y de 31 personas sanas no consumidoras de alcohol, emparejadas por sexo, edad, lateralidad y nivel socioeconómico, pero no por años de estudio (Tabla 1). Recopilamos datos sociodemográficos de los historiales clínicos registrados por personal sanitario y verificados por un evaluador. Las personas con dependencia al alcohol fueron reclutadas cuando comenzaron tratamiento psicosocial en la Unidad de Conductas Adictivas del Hospital Nostra Senyora de Meritxell (Andorra). Los criterios de inclusión fueron los criterios del DSM-IV para dependencia al alcohol, y abstinencia mínima de 15 días antes de las pruebas, confirmado mediante los análisis de orina dos veces por semana (véase Tabla 1 para datos descriptivos sobre cantidad y duración de uso de drogas). Los criterios de exclusión incluyeron un diagnóstico actual o historia de otro abuso/dependencia de psicoestimulantes (exceptuando la nicotina), diagnóstico de comorbilidad de trastornos de los Ejes I o II, historial de lesiones cerebrales o trastornos neurológicos, y déficits cognitivos severos provocados por la demencia. Los participantes del grupo de control fueron reclutados mediante comunicación de boca a boca entre adultos que residían en la misma zona geográfica que los pacientes. Además de los criterios anteriores, los controles no podían haber sido diagnosticados anteriormente con abuso o dependencia a sustancias, excluyendo el consumo de alcohol social (menos de 10 unidades semanales) actual o en el pasado, y el uso de nicotina.

Tabla 1. Datos sociodemográficos y de uso de alcohol en personas con dependencia al alcohol y en personas del grupo de control.

\begin{tabular}{|c|c|c|c|c|}
\hline & $\begin{array}{r}\text { Personas con } \\
\text { dependencia al alcohol } \\
\text { Media (SD) }\end{array}$ & $\begin{array}{r}\text { Grupo de control } \\
\text { Media (SD) }\end{array}$ & $\mathrm{t} / \chi^{2}$ & Valor $p$ \\
\hline \multicolumn{5}{|l|}{ Variables sociodemográficas } \\
\hline Edad & $52,53(5,79)$ & $48,65(10,89)$ & $-1,69$ & 0,097 \\
\hline Sexo & 31 (Hombres) & 28 (Hombres) & & \\
\hline Lateralidad & 29 (Diestro) / 2 (Zurdo) & 26 (Diestro) / 2 (Zurdo) & 0,01 & 0,916 \\
\hline Años de estudio & $13,71(2,09)$ & $17,22(2,86)$ & 5,34 & $<0,001$ \\
\hline Nivel socioeconómico & $8(B) / 17(M) / 3(A)$ & $5(B) / 21(M) / 5(A)$ & 1,47 & 0,481 \\
\hline \multicolumn{5}{|l|}{ Variables de uso de alcohol } \\
\hline Abstinencia de alcohol (meses) & $2,42(14,02)$ & & & \\
\hline Consumo de alcohol mensual (UBE) & $562,70(419,02)$ & $21,47(13,09)$ & $-7,20$ & $<, 001$ \\
\hline Duración del consumo de alcohol (años) & $27,63(8,19)$ & $21,20(9,69)$ & $-2,70$ & 0,009 \\
\hline Consumo de alcohol total (UBE) & $197692(168924)$ & $5203(4507)$ & $-6,35$ & $<, 001$ \\
\hline
\end{tabular}

Nota. Consumo de alcohol total (a lo largo de la vida) como producto de la cantidad y duración medias; SD: desviación estándar; B: bajo; M: medio; A: alto; UBE: unidades de bebida estándar. 


\section{Instrumentos}

Estimulos emocionales. Una serie de 30 imágenes fueron cogidas del Sistema Internacional de Imágenes Afectivas (IAPS; Lang, Öhman y Vaitl, 1988) para su uso como estímulo. De acuerdo con los valores normativos españoles (Moltó et al., 2013), definimos las tres categorías de imágenes: (i) neutra (10 imágenes mostrando paisajes y objetos de la vida cotidiana), (ii) agradable (10 imágenes mostrando escenas de naturaleza sexual o de deporte excitantes), y (iii) desagradable (10 imágenes mostrando fallecimientos o mutilaciones como resultado de accidentes). Los códigos de las imágenes seleccionadas fueron: 4658, 4669, 4670, 4672, 4687, $5621,8178,8179,8186$, y 8496 para agradable; 1525, 3000, 3062, 3068, 3080, 3150, 3250, 9301, 9405, y 9635 para desagradable; y 7004, 7009, 7041, 7175, 7185, 7187, 7224, 7233, 7705 y 7950 para neutra. Los participantes puntuaron su experiencia emocional para valencia (de 1 -desagradable- a 9 -agradable), activación (de 1 -relajado-a 9 -excitado-), y dominancia (de 1 -dominante-a 9-dominado-) usando el Maniquí de Autoevaluación (SAM; Bradley y Lang, 1994). Usamos software E-Prime (Psychological Tools, EE.UU.) para controlar los tiempos y la presentación de los estímulos y para recopilar las valoraciones subjetivas.

Medidas autoinformadas. Además del SAM, los participantes cumplimentaron los siguientes instrumentos: (i) Entrevista Clínica Estructurada para DSM-IV (SCID-I; First, 2007); (ii) Entrevista para la evaluación de la conducta adictiva (IRAB; Verdejo-García, López-Torrecillas, Aguilar de Arcos y Pérez-García, 2005); (iii) Inventario clínico multiaxial de Millon III (MCMI-III; Millon y Davis, 1997); (iv) Inventario de síntomas, revisado (SCL-90-R; Derogatis, 1977); (v) Mini examen del estado mental (MMSE; Folstein, Folstein y McHugh, 1975); y (vi) Escala de impulsividad de Barratt (BIS-11; Patton, Stanford y Barratt, 1995). Los cinco primeros instrumentos se usaron para valorar el grado de cumplimiento de los criterios de inclusión/exclusión. La BIS-11, una medida de rasgos de impulsividad, contiene 30 ítems que valoran las manifestaciones de impulsividad que los participantes puntúan en términos de frecuencia: nunca o rara vez; de vez en cuando; a menudo; siempre o casi siempre (con puntuaciones entre 0 y 4). La puntuación total en impulsividad y las puntuaciones de las tres subescalas (impulsividad cognitiva, motora y no planeada) fueron recogidas como medidas dependientes. La BIS-11 ha mostrado una alta fiabilidad para valorar conductas de riesgo (Alfa de Cronbach = ,83, Stanford et al., 2009). Por último, se calculó el consumo total de alcohol a lo largo de la vida como producto de la cantidad y duración medias de uso de alcohol.

\section{Recopilación y tratamiento de datos psicofisiológicos}

La CP y la TC se registraron con el instrumento Biopac MP150 (Biopac Systems Inc., EE.UU.). La TC, en latidos por minuto (lat/min), se cogió del electrocardiograma (ECG) grabado a $2000 \mathrm{~Hz}$. Se ubicaron electrodos ECG (Ag/AgCl) a los tobillos derecho e izquierdo de los participantes, y a la muñeca de la mano no-dominante, basado en la derivación II de Einthoven. Se calculó la TC con software AcqKnowledge 3.9.1, y se editó los artefactos (en su caso) mediante interpolación lineal. La CP en micro-Siemens $(\mu \mathrm{S})$ se registró a una velocidad de muestreo de $30 \mathrm{~Hz}$ con electrodos $\mathrm{Ag}-\mathrm{AgCl}$ con crema electrólito inerte $0.05 \mathrm{M} \mathrm{NaCl}$, sobre la superficie palmar de los falanges segundo y tercero de la mano no-dominante de cada participante. Con el fin de extraer el patrón de respuesta de la TC $(\Delta \mathrm{HR})$ y $\mathrm{CP}(\Delta \mathrm{SC})$, obtuvimos los valores $0.5 \mathrm{~s} \times 0.5 \mathrm{~s}$ durante los primeros $6 \mathrm{~s}$ después de la presentación de la imagen (12 valores), expresados como puntuaciones diferenciales respecto de la media obtenida durante los $3 \mathrm{~s}$ previos a la presentación de la imagen (línea basal).

\section{Trámites previos al procedimiento}

Antes de realizar las pruebas, todos los participantes firmaron un formulario de consentimiento y realizaron una valoración inicial usando las medidas de autoevaluación descritas anteriormente. Después de registrar sus respuestas fisiológicas durante 5 min en estado de reposo (línea basal), se mostró a los participantes la serie de imágenes mientras se les registró sus datos fisiológicos. Las diversas categorías de imágenes (neutra, agradable y desagradable) se presentaron en orden equilibrado a todos los participantes. Antes de visualizar cada imagen, aparecía en pantalla una cruz blanca durante $3 \mathrm{~s}$ para ayudar a los participantes a fijar la visa en la pantalla. Después, las imágenes fueron expuestas durante $6 \mathrm{~s}$, seguidas de una pantalla en negro durante $10 \mathrm{~s}$ como intervalo entre los estímulos. Se pidió a los participantes que se fijasen en cada imagen durante todo el tiempo que aparecía en pantalla. Después de quitar los electrodos, los participantes de nuevo observaron las imágenes para puntuarlas, usando la versión informatizada del SAM, sin límite de tiempo. El estudio fue aprobado por el Comité de Ética de estudios humanos de la Universidad de Granada (España) y el Comité de Ética para estudios clínicos del Hospital Nostra Senyora de Meritxell.

\section{Análisis estadístico}

Los grupos tenían diferentes niveles de años de estudio (Tabla 1) y por ello se incluyó este variable como covariable en el análisis de los instrumentos autoinformados. Las diferencias en impulsividad entre los grupos se sometieron a prueba usando las ANCOVA monofactoriales. Las valoraciones subjetivas de valencia, activación y dominancia se analizaron usando ANCOVA mixtas de 2 (Grupo: dependencia al alcohol y controles sanos) $\times 3$ (Categoría de imagen: neutra, agradable y desagradable). Las medidas fisiológicas tónicas (línea basal durante el periodo de 5 minutos de reposo) se analizaron con pruebas t independientes. Los grupos tenían diferencias en la CP [depen- 
dencia al alcohol 5,16 $\pm 2,57 \mu \mathrm{S}$; controles $3,72 \pm 1,96 \mu \mathrm{S} ; \mathrm{t}=$ $-2,34, p=, 024$ ] y la TC [dependencia al alcohol 77,45 $\pm 13,09$ lat/min; controles 70,15 $\pm 12,13 \mathrm{lat} / \mathrm{min} ; \mathrm{t}=-2,21, \mathrm{p}=, 031]$ $\mathrm{y}$, por tanto, incluimos dichas variables como covariables en los respectivos análisis fisiológicos. $\Delta \mathrm{SC}$ y $\Delta \mathrm{HR}$ durante la visualización de las imágenes fueron analizadas usando 2(3×12) ANCOVA mixtas, con un factor entre-sujetos (grupo) y dos factores de medidas repetidas [Categoría de imagen y Patrón de respuesta (los $120.5 \times 0.5 \mathrm{~s}$, valores posteriores a los estímulos)]. Usamos el procedimiento Huynh-Feldt para ajustar para grados de libertad en el análisis de las medidas repetidas. Presentamos los resultados con los grados de libertad originales y los valores p corregidos.

Implementamos un análisis de regresión logística paso a paso para valorar los efectos de uso de alcohol y de impulsividad sobre la experiencia emocional. Incluimos los picos de las respuestas fisiológicas durante la visualización de las imágenes como variables dependientes: subidas de la CP (valor máximo - línea basal) y caídas de la TC (valor mínimo - línea basal). Las variables predictoras fueron consumo de alcohol total y puntuación total de impulsividad de la BIS. No incluimos las puntuaciones de las tres subescalas de la BIS por su alta correlación con impulsividad total (cognitiva: $\mathrm{r}=, 69$; motora: $\mathrm{r}=, 85$; no planeada: $\mathrm{r}=, 88$; todos $\operatorname{los} \mathrm{p}<, 001)$.

\section{Resultados}

\section{Tasa Cardiaca}

La interacción entre las tres dimensiones Grupo $\times$ Categoría de imagen x Patrón de respuesta $[\mathrm{F}(22,1232)=2,73, p$ $\left.=, 024, \eta_{p}^{2}=, 046\right]$ fue significativa. El análisis de la interacción entre Categoría de imagen x Patrón de respuesta en cada grupo mostró efectos significativos para los Controles [F(22, $\left.660)=2,79, p=, 032, \eta_{p}^{2}=, 085\right]$, pero no para los participan- tes con dependencia al alcohol $[\mathrm{F}(22,594)=1,14, p=, 343$, $\left.\eta_{p}^{2}=, 040\right]$. En el grupo de control, la respuesta de la TC fue significativa para imágenes agradables $[\mathrm{F}(11,330)=5,29, p=$ $\left., 010, \eta_{p}^{2}=, 150\right]$ y desagradables $[\mathrm{F}(11,330)=3,55, p=, 021$, $\left.\eta_{p}^{2}=, 106\right]$, pero no para la categoría neutra $[\mathrm{F}(11,330)=1,18$, $\left.p=, 317, \eta_{p}^{2}=, 038\right]$. En el grupo con dependencia al alcohol, la respuesta de la TC no fue significativa para ninguna de ambas categorías de imagen. Como muestra la Figura 1 (parte superior), en el grupo de control, las imágenes agradables indujeron una aceleración de la TC inicial de hasta 2,5 s seguido de una deceleración pronunciada que se mantuvo durante la presentación de imágenes íntegra [tendencia cuadrática para el periodo: $\left.\mathrm{F}(1,30)=5,34, p=, 028, \eta_{p}^{2}=, 151\right]$. La mejor descripción del patrón de respuesta a imágenes desagradables es una deceleración de la TC, aunque de menor magnitud que la asociada a las imágenes agradables [con únicamente la tendencia lineal significativa: $\mathrm{F}(1,30)=6,65$, $\left.p=, 015, \eta_{p}^{2}=, 181\right]$.

\section{Conductancia de la piel}

La interacción entre Grupo $\times$ Categoría de imagen x Patrón de respuesta fue significativa $[\mathrm{F}(22,1232)=3,74, p=$ $\left., 039, \eta_{p}^{2}=, 051\right]$. El patrón de respuesta de la CP difirió según la categoría de imagen en el grupo de control [Categoría de imagen x Patrón de respuesta: $\mathrm{F}(22,660)=5,33, p=, 003$, $\left.\eta_{p}^{2}=, 165\right]$ pero no en el grupo con dependencia al alcohol $\left[\mathrm{F}(22,594)=2,18, p=, 106, \eta_{p}{ }^{2}=, 080\right]$. El grupo de control tuvo una respuesta significativa de la CP para imágenes agradables $\left[\mathrm{F}(11,330)=3,94, p=, 040, \eta_{p}^{2}=, 127\right]$, pero no para las otras categorías de imagen. Como muestra la Figura 1 (parte inferior), subidas en la CP ocurrieron ante imágenes agradables para el grupo de control entre 3 y $6 \mathrm{~s}$, con el pico de la respuesta a los $5 \mathrm{~s}$ [tendencias cúbicas: $\mathrm{F}(1,30)=4,32$, $\left.p=, 047, \eta_{p}^{2}=, 138\right]$. En el grupo con dependencia al alcohol, no se obtuvieron respuestas significativas para CP.

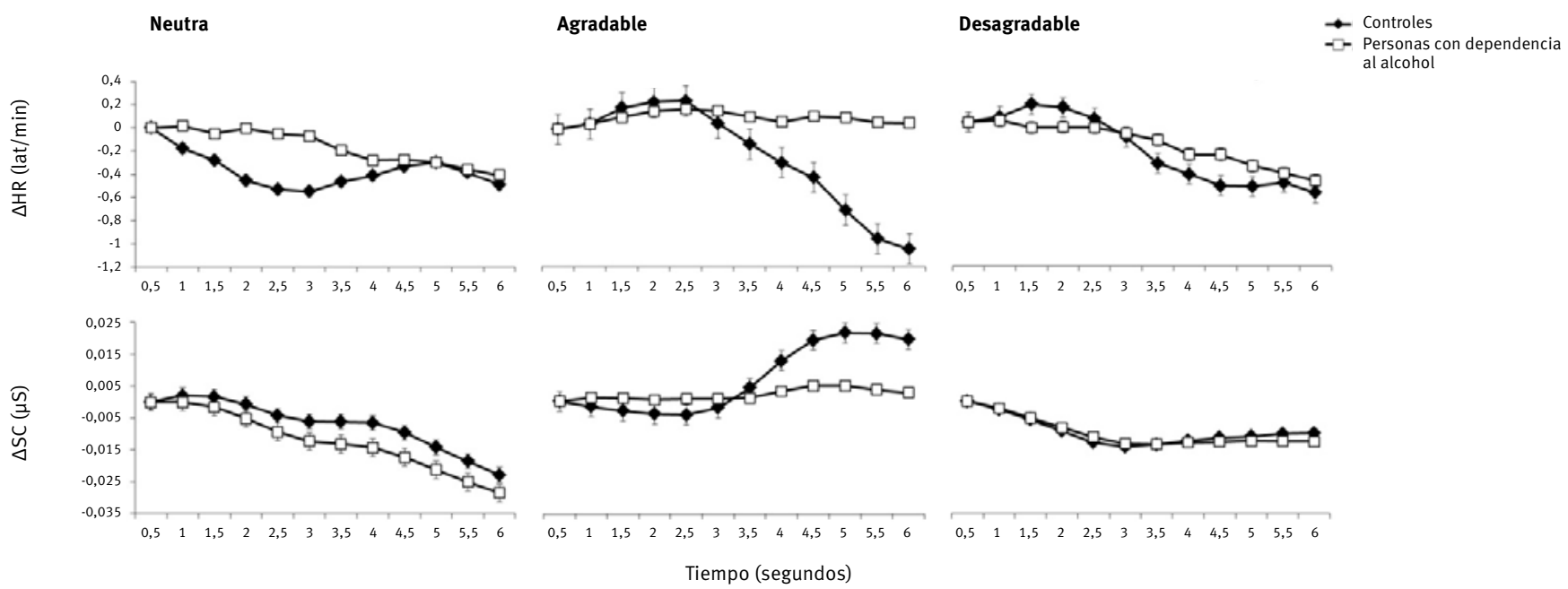

Figura 1. Tasa cardiaca (parte superior) y conductancia de la piel (parte inferior) durante la visualización de imágenes en función de Categoría de imagen (Neutra, Agradable y Desagradable), y Grupo (Controles y personas con dependencia al alcohol). 


\section{Valoraciones subjetivas de valencia, activación y dominancia (SAM)}

No hubo interacciones significativas de Grupo $\times$ Categoría de imagen en términos de valoraciones subjetivas de valencia $\left[\mathrm{F}(2,112)=, 60, p=, 545, \eta_{p}^{2}=.011\right]$, activación $[\mathrm{F}(2$, $\left.112)=1,20, p=, 306, \eta_{p}^{2}=, 021\right]$ o dominancia $[\mathrm{F}(2,122)=$ ,04, $\left.p=, 936, \eta_{p}^{2}=, 001\right]$. El efecto principal de Grupo no fue estadísticamente significativo para ninguna dimensión (todas las $\mathrm{F}(1,56)>1,65$, todas las $p>.2$, todas las $\mathrm{s}<.03)$. El efecto de Categoría de imagen fue significativo para valencia $\left[\mathrm{F}(2,112)=10,20, p<, 001, \eta_{p}^{2}=, 154\right]$ y activación $[\mathrm{F}(2$, $\left.112)=3.56, p=, 032, \eta_{p}^{2}=, 060\right]$, pero no para dominancia $\left[\mathrm{F}(2,112)=1,61, p=, 208, \eta_{p}^{2}=, 028\right]$. Ambos grupos mostraron las diferencias esperadas en valoraciones de valencia y activación entre las Categorías de imagen diferentes, y aunque la diferencia en dominancia no fue significativa, según los valores normativos (Moltó et al., 2013) las imágenes desagradables resultaron en puntuaciones en dominancia más bajas que todas las demás condiciones (Tabla 2 y Figura 2).

\section{Rasgos de personalidad impulsiva (BIS-11)}

Los participantes con dependencia al alcohol mostraron niveles más altos de impulsividad total $[\mathrm{F}(2,56)=21,26, p<$ ,001, $\left.\eta_{p}^{2}=, 432\right]$ y de impulsividad en las tres subescalas de impulsividad [Cognitiva: $\mathrm{F}(2,56)=6,38, p=, 003, \eta_{p}^{2}=, 186$; Motora: $\mathrm{F}(2,56)=20,51, p<, 001, \eta_{p}^{2}=, 423$; y no planeada $\left.\mathrm{F}(2,56)=9,62, p<, 001, \eta_{p}^{2}=, 256\right]$ que los participantes en el grupo de control. En la muestra íntegra, la impulsividad total tuvo una asociación positiva con el consumo de alcohol total $(r=, 63, p<, 001)$. La impulsividad total tuvo una correlación positiva con el consumo de alcohol total en participantes con dependencia al alcohol $(r=, 44, p<$ ,022), pero no en los controles $(r=-, 05, p<, 805)$.

\section{El impacto de la impulsividad y el uso de alcohol en las respuestas fisiológicas}

La impulsividad total predijo subidas en la CP para la muestra general durante la presentación de imágenes agradables $[\mathrm{F}(1,57=4,90, \mathrm{p}=, 031]$ y desagradables $[\mathrm{F}(1$, $57)=6,66, p=, 012]$, mientras que el consumo de alcohol no contribuyó a los modelos predictivos. Hubo una asociación inversa con las subidas en la CP durante la presentación de imágenes agradables $(\beta=-.281)$ y desagradables ( $\beta=-.323$ ), explicando el 6,3\% para imágenes agradables y el $8,9 \%$ para imágenes desagradables de la varianza total (con el R2 ajustado). El análisis de regresión durante la presentación de imágenes agradables, para ambos grupos por separado, mostró que la impulsividad total fue un predictor significativo sólo para los participantes con dependencia al alcohol $[\mathrm{F}(1,26)=8,13, \mathrm{p}=, 008]$ y no para los controles. En el grupo con dependencia al alcohol, la impulsividad total tuvo una relación inversa con las subidas en la CP durante la presentación de imágenes agradables ( $\beta=-.488$ ), explicando el $20,9 \%$ de la varianza en las res-

Tabla 2. Media y desviación estándar (entre paréntesis) de las valoraciones subjetivas de valencia, activación y dominancia evocadas por las tres Categorías de Imágenes para personas con dependencia al alcohol y participantes del grupo de control.

\begin{tabular}{|c|c|c|c|c|c|c|}
\hline \multirow{2}{*}{$\begin{array}{l}\text { Valoraciones } \\
\text { subjetivas }\end{array}$} & \multicolumn{3}{|c|}{ Personas con dependencia al alcohol } & \multicolumn{3}{|c|}{ Participantes del grupo de control } \\
\hline & Neutra & Agradable & Desagradable & Neutra & Agradable & Desagradable \\
\hline Valencia & $5,50(, 61)^{\star}$ & $7,24(1,29)^{\star}$ & $1,97(, 54)^{\star}$ & $5,75(, 93)^{*}$ & $7,54(1,05)^{\star}$ & $1,86(, 60)^{\star}$ \\
\hline Activación & $4,53(1,05)^{\star}$ & $5,93(1,66)^{\star}$ & $7,45(, 93)^{\star}$ & $3,74(1,43)^{\star}$ & $5,72(1,86)^{\star}$ & $7,52(, 81)^{\star}$ \\
\hline Dominancia & $8,48(, 98)$ & $7,66(1,76)$ & $5,79(2,60)$ & $8,51(, 84)$ & $7,63(1,19)$ & $5,95(2,25)$ \\
\hline
\end{tabular}

Nota. * Diferencias significativas entre categorías de imágenes (valor $\mathrm{p}<, 05$ ).
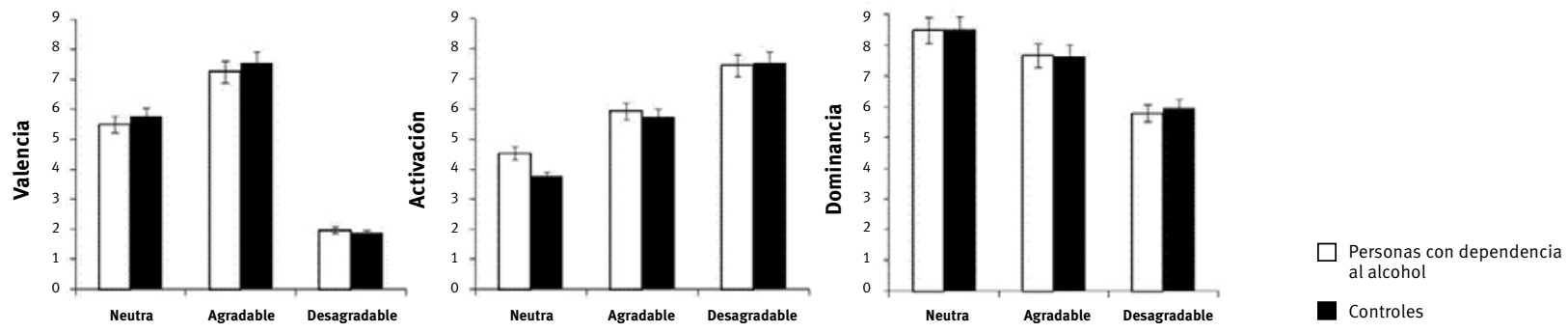

Figura 2. Valoraciones subjetivas de valencia, activación y dominancia ante imágenes agradables, desagradables y neutras en personas con dependencia al alcohol y participantes del grupo control. Las barras son el error estándar de la media. 
Tabla 3. Capacidad predictiva de la puntuación total en la Escala de Impulsividad de Barratt en la respuesta de conductancia de la piel (resultados significativos en el análisis de regresión múltiple por pasos).

\begin{tabular}{lllll}
\hline Variable dependiente & $\beta$ & $\mathrm{r}^{2}$ & $\mathrm{~F}$ & $\mathrm{p}$ \\
\hline Agradable (muestra total) & -.28 & .06 & 4.90 & .031 \\
Desagradable (muestra total) & -.32 & .09 & 6.66 & .012 \\
$\begin{array}{l}\text { Agradable (personas con } \\
\text { dependencia al alcohol) }\end{array}$ & -.49 & .21 & 8.13 & .008 \\
$\begin{array}{l}\text { Desagradable (personas con } \\
\text { dependencia al alcohol) }\end{array}$ & -.32 & .10 & 2.99 & .096 \\
\hline
\end{tabular}

Nota. Valor $r^{2}$ ajustado y valor $\beta$ estandarizado.

puestas de la CP (Tabla 3). El análisis de regresión jerárquica, en el cual el consumo de alcohol total se incluyó en el bloque 1 y la impulsividad total en el bloque 2, el efecto de la impulsividad sobre las subidas en la CP durante la presentación de imágenes agradables siguió siendo significativa $[\mathrm{F}(2,25)=4,26, \beta=-, 424, \mathrm{p}=, 026]$. Para imágenes desagradables, la impulsividad total tuvo una asociación marginal con las subidas en la CP en los participantes con dependencia al alcohol $[\mathrm{F}(1,26)=2,99, \beta=-, 321, \mathrm{p}=, 096]$ y para los participantes en el grupo de control $[\mathrm{F}(1,29)=$ $3,99, \beta=-, 348, p=, 055]$. El consumo de alcohol no fue un predictor significativo en ningún modelo. No encontramos ningún modelo de regresión significativo para la predicción de respuestas en TC.

\section{Discusión}

Este estudio tuvo como objetivo estudiar las reacciones psicofisiológicas y subjetivas ante estímulos relevantes cotidianos (no relacionados con las sustancias) en personas con dependencia al alcohol con el fin de determinar las asociaciones con la impulsividad y el nivel de consumo de alcohol. Respecto del primer objetivo, aunque no hallamos diferencias entre los grupos en las valoraciones subjetivas de imágenes emocionales, los hallazgos muestran que la reactividad de la TC y la CP es más disminuida en personas con dependencia al alcohol comparado con los participantes en el grupo de control. El grupo de personas con dependencia al alcohol no mostró deceleración de la TC ante imágenes emocionalmente positivas y negativas, ni la subida de la CP ante imágenes emocionalmente positivas que mostró el grupo de control. Estos resultados sugieren que las personas con dependencia al alcohol tienen respuestas fisiológicas disminuidas ante estímulos emocionales cotidianos, positivos y negativos.

La ausencia de reacciones en CP y TC ante imágenes positivas está en línea con la propuesta de una desregulación de los mecanismos de refuerzo en la adicción (Robinson y Berridge, 2001, 2003). La reactividad de la CP y la TC debería verse atenuada por el predominio del alcohol en el sistema de refuerzos, que sustituiría el valor motivacional de los refuerzos naturales mostrados en las imágenes (Robinson y Berridge, 2001, 2003). Subidas de la CP están asociadas con la experiencia de emociones excitantes (Barry et al., 2004, 2006; Lang y Davis, 2006). Podría explicarse la ausencia de una respuesta identificable de la CP ante estímulos positivos en personas con dependencia al alcohol por la prominencia consolidada de los estímulos relacionados con las sustancias en sus sistemas motivacionales, junto con la relevancia disminuida de los refuerzos naturales (Robinson y Berridge, 2001, 2003; Verdejo-García, Bechara, Recknor y Perez-Garcia, 2006). Estos hallazgos son coherentes con los resultados de estudios con personas adictas a los opioides (Aguilar de Arcos et al., 2008) y personas adictas al juego (Lole et al., 2012), que han mostrado reacciones de activación disminuida (CP baja) ante refuerzos naturales. También podría interpretarse la ausencia de deceleración de la TC ante imágenes positivas en los participantes con dependencia al alcohol como indicador de la baja prominencia de las motivaciones, mostrando una atención disminuida ante los refuerzos naturales (no relacionados con las sustancias) (Robinson y Berridge, 2001, 2003; Verdejo-García et al., 2006).

La ausencia de reacción de la TC ante imágenes negativas en personas con dependencia al alcohol, como se observó en los participantes del grupo de control, está en línea con la hipótesis de los marcadores somáticos en la adicción (Verdejo-García y Bechara, 2009). Esta hipótesis mantiene que las personas con dependencia al alcohol mostrarían dificultades en generar señales emocionales adversas asociadas a una respuesta fisiológica (Verdejo-García y Bechara, 2009), lo que, a su vez, puede resultar en una reacción disminuida de la TC ante imágenes negativas. La visualización pasiva de imágenes emocionalmente negativas se asocia, en general, con una deceleración de la TC (Danko, Gracheva, Boytsova y Solovjeva, 2011; Lang et al., 1993; Palomba et al., 2000). La ausencia de una disminución de la TC en respuesta a estímulos negativos en personas con dependencia al alcohol está en línea con estudios que han encontrado una reactividad de la TC disminuida ante estímulos emocionalmente negativos, incluyendo imágenes negativas (Gerra et al., 2003), y ante las consecuencias negativas de violaciones de la moralidad (Carmona-Perera et al., 2013).

Por tanto, la ausencia de una deceleración en personas con dependencia al alcohol podría reflejar una implicación deficiente con las emociones de valencia negativa durante la visualización de imágenes negativas, y una baja prominencia de las motivaciones de los refuerzos naturales durante la visualización de imágenes positivas. Estos resultados son coherentes con estudios sobre los sesgos de atención hacia los estímulos afectivos en la adicción; estos estudios han mostrado que las señales relacionadas con la adicción son objeto de mayor atención, y que los estímulos 
cotidianos positivos tienen baja prominencia motivacional en personas con adicción, incluyendo las personas con adicción al juego (Hudson , Jacques y Stewart, 2013), usuarios de opioides (Lubman, Peters, Mogg, Bradley y Deakin, 2000; Robbins y Ehrnan, 2004), y personas con dependencia al alcohol (Garfield, Allen, Cheetham, Simmons y Lubman, 2015; Lambe, Hudson y Stewart, 2015).

Observamos una disociación entre las respuestas fisiológicas y subjetivas en personas con dependencia al alcohol en este estudio, con respuestas autonómicas disminuidas e informes subjetivos normales respecto de los estímulos emocionales. Otros estudios sobre personas con adicción expuestas a paradigmas de reactividad emocional también han informado esta disociación entre la reactividad fisiológica y las respuestas emocionales subjetivas (Aguilar de Arcos et al., 2008; Gerra et al., 2003). Dicha disociación podría interpretarse en el contexto de alteraciones en la sensibilidad interoceptiva del alcoholismo y otras conductas de uso de sustancias, dado que los efectos estimulantes de las drogas sobre el sistema nervioso autonómico podrían alterar las percepciones de los estados corporales (Naqvi y Bechara, 2010; Paulus, Feinstein, Simmons y Stein, 2004; Verdejo-García, Clark y Dunn, 2012). De manera específica, los usuarios de alcohol han mostrado una percepción disminuida de las señales corporales, según medidas de las tareas de seguimiento de latidos cardíacos (Schmidt, Eulenbruch, Langer y Banger, 2013). Estos resultados sugieren deficiencias para generar y percibir los correlatos fisiológicos de las emociones en personas con dependencia al alcohol, que podría originar de la dominancia de las señales relacionadas con las drogas (Dunn et al., 2010; Naqvi y Bechara, 2010; Verdejo-García et al., 2012).

Nuestro segundo objetivo fue determinar la contribución de la impulsividad en la capacidad de respuesta emocional. Primero, las personas con dependencia al alcohol mostraron niveles más altos de impulsividad: esto se observó en los tres dominios medidos por la escala BIS: cognitiva, motora, y no planeada, en línea con estudios anteriores (Bowden-Jones, Phillips, Rogers, Hutton y Joyce 2005; Körner, Schmidt y Soyka, 2015; Petry, 2001). La impulsividad fue predictora de respuestas disminuidas de la CP ante estímulos emocionales positivos y negativos en personas con dependencia al alcohol, pero no fue predictora de respuestas de TC. Aunque hubo una asociación entre la impulsividad total y el consumo de alcohol total en personas con dependencia al alcohol, el consumo de alcohol no fue predictor de las respuestas de la CP o de la TC. Además, tras controlar por el consumo de alcohol total, la impulsividad continúa siendo un predictor significativo de subidas en la CP ante imágenes agradables en personas con dependencia al alcohol. Este resultado sugiere que el consumo de alcohol no es un factor mediador relevante en la relación entre impulsividad y respuestas de la CP. Aunque son necesarios estudios futuros para explorar dichas asociaciones en mayor profundidad, estos resultados sugieren que la impulsividad puede ser un factor crítico para explicar las respuestas fisiológicas disminuidas ante imágenes emocionales en personas con dependencia al alcohol, con independencia del consumo de alcohol total.

La asociación entre impulsividad y respuestas fisiológicas disminuidas puede subyacer la tendencia en la adicción a un comportamiento precipitado durante emociones significativamente positivas o negativas (Cyders y Smith, 2008). Este rasgo de urgencia influye en los procesos atencionales y puede resultar de las diminuciones en atención a estímulos emocionales (Settles, Zapolski y Smith, 2014; Smith y Cyders, 2016). Existe apoyo claro de que este rasgo de urgencia es un predictor del inicio y de aumentos en el uso de sustancias, incluyendo la ingesta de alcohol (Cyders et al., 2010; Kaiser, Bonsu, Charnigo, Milich y Lynam, 2016). Nuestros resultados también son similares a otros estudios fisiológicos sobre adicción al juego, que mostraron que las respuestas deficientes de la CP estaban relacionadas con la impulsividad y la toma de decisiones desfavorable, caracterizada por déficits en predecir las consecuencias negativas a largo plazo de la conducta (Bechara y Damasio, 2002; Dom, Wilde, Hulstijn, Brink y Sabbe, 2006; Fein, Klein y Finn, 2004; Fishbein et al., 2005). Conjuntamente, estos resultados amplían la evidencia previa respecto de la relación entre variables de personalidad impulsivas y alteraciones en el autocontrol y la regulación emocional en personas que abusan de las sustancias (Brenner, Beauchaine y Sylvers, 2005; Clark, Cornelius, Kirisci y Tarter, 2005; Carou et al., 2017; Nigg, Hinshaw y Huang-Pollack, 2006; Ohannessian y Hesselbrock, 2007, Verdejo-García et al., 2007), al mostrar la existencia de una asociación entre impulsividad y respuestas fisiológicas disminuidas ante estímulos naturales y emocionalmente significativos en personas con dependencia al alcohol.

Nuestros resultados han de ser interpretados en el contexto de ciertas limitaciones metodológicas. Primero, los grupos no estaban emparejados por nivel de estudios. Para abordar dicha limitación, incluimos años de estudio como un covariable en el análisis de las medidas autoinformadas. Segundo, dado el diseño transversal del estudio, los datos actuales no pueden determinar causalidad; esto podría abordarse en estudios futuros con modelos de vulnerabilidad ante el consumo de drogas (Verdejo-García y Bechara, 2009). Tercero, implementamos únicamente una medida autoinformada de impulsividad y no aplicamos una tarea de rendimiento; esto podría incluirse en estudios futuros. No obstante, las medidas de impulsividad subjetivas pueden ser más adecuadas como índice del aspecto social del comportamiento impulsivo, omitido por las medidas neurofisiológicas (Moeller, Barratt, Dougherty, Schmitz y Swann, 2001). Otra limitación es la ausencia de medición de algunos moderadores potenciales del vínculo entre el procesamiento de las emociones y la impulsividad -e.g., 
rasgos de personalidad (Muller, Weijers, Boning y Wiesbeck, 2008) - o del sesgo de deseabilidad hacia las demandas sociales y experimentales (Hess y Kotter-Grühn, 2011; Najström y Jansson, 2006). Estudios futuros han de aportar mayor claridad a las asociaciones entre la reactividad fisiológica a los estímulos emocionales y la impulsividad, y a los factores moderadores potenciales.

En resumen, las respuestas disminuidas de la TC y la CP a estímulos emocionales cotidianos podría ser un rasgo disfuncional predominante en las personas con dependencia al alcohol. En el caso de la CP, esta respuesta disminuida estaba asociada con la impulsividad. Estos resultados han mostrado déficits en las respuestas autonómicas ante estímulos naturales y emocionalmente significativos en personas con dependencia al alcohol, y aportan pruebas iniciales de la contribución de la impulsividad a los déficits en el procesamiento emocional de esta población. Estos resultados pueden tener implicaciones importantes para las intervenciones relacionadas con el alcohol, que deberían poner el foco en los rasgos de impulsividad y restaurar la prominencia de los estímulos cotidianos para potencialmente mejorar el procesamiento de las emociones.

\section{Conflicto de intereses}

Los autores declaran la inexistencia de conflicto de intereses.

\section{Referencias}

Aguilar de Arcos, F., Verdejo-García, A., Ceverino, A., Montañez-Pareja, M., López-Juárez, E., Sánchez-Barrera, M., ...Pérez-García, M. (2008). Dysregulation of emotional response in current and abstinent heroin users: negative heightening and positive blunting. Psychopharmacology, 198, 159-166. doi:10.1007/s00213-008-1110-2.

Allsop, S., Saunders, B. y Phillips, M. (2000). The process of relapse in severely dependent male problem drinkers. Addiction, 95, 95-106. doi:10.1046/j.13600443.2000.9519510.x.

Barry, R. J. (2006). Promise versus reality in relation to the unitary orienting reflex: a case study examining the role of theory in psychophysiology. International Journal of Psychophysiology, 62, 353-366. doi:10.1016/j.ijpsycho.2006.01.004.

Barry, R. J., Clarke, A. R., McCarthy, R., Selikowitz, M., Rushby, J. A. y Ploskova, E. (2004). EEG differences in children as a function of resting-state arousal level. Clinical Neurophysiology, 115, 402-408. doi:10.1016/S13882457(03)00343-2.

Beck, A., Wustenberg, T., Genauck, A., Wrase, J., Schlagenhauf, F., Smolka, M. N., Mann, K. y Heinz, A. (2012). Effect of Brain Structure, Brain Function, and Brain Connectivity on Relapse in Alcohol-Dependent Patients.
Archives of General Psychiatry, 69, 842-853. doi:10.1001/ archgenpsychiatry.2011.2026.

Bechara, A. (2005). Decision making, impulse control and loss of willpower to resist drugs: a neurocognitive perspective. Nature Neuroscience, 8, 1458-1463. doi:10.1038/ nn1584.

Bechara, A. y Damasio, A. (2002). Decision-making and addiction (part I): impaired activation of somatic states in substance dependent individuals when pondering decisions with negative future consequences. Neuropsychologia, 40, 1675-1689. doi:10.1016/S0028-3932(02)00015-5.

Bjork, J. M., Hommer, D. W., Grant, S. J. y Danube, C. (2004). Impulsivity in abstinent alcohol-dependent patients: relation to control subjects and type 1/type 2 like traits. Alcohol, 34, 133-150. doi:10.1016/j.alcohol.2004.06.012.

Bowden-Jones, H., Phillips, M., Rogers, R., Hutton, S. y Joyce, E. (2005). Risk-taking on tests sensitive to ventromedial prefrontal cortex dysfunction predicts early relapse in alcohol dependency: a pilot study. Journal of Neuropsychiatry E Clinical Neurosciences, 17, 417-420. doi:10.1176/ jnp.17.3.417.

Bradley, M. M. y Lang P. J. (1994). Measuring emotion: the Self-Assessment Manikin and the Semantic Differential. Journal of Behavior Therapy and Experimental Psychiatry, 25, 49-59. doi:10.1016/0005-7916(94)90063-9.

Brenner, S. L., Beauchaine, T. P. y Sylvers, P. D. (2005). A comparison of psychophysiological and self-report measures of BAS and BIS activation. Psychophysiology, 42, 108115. doi:10.1111/j.1469-8986.2005.00261.x.

Breversa, D., Noëla, X., Ermerb, E., Dabiria, D., Verbancka, P. y Kornreich, C. (2013). Unfairness sensitivity and social decision-making in individuals with alcohol dependence: A preliminary study. Drug and Alcohol Dependence, 133, 772-775. doi.10.1016/j.drugalcdep.2013.08.013.

Carmona-Perera, M., Clark, L., Young, L., Pérez-García, M. y Verdejo-García, A. (2014). Impaired decoding of fear and disgust predicts utilitarian moral judgment in alcohol-dependent individuals. Alcohol Clinical and Experimental Research, 38, 179-185. doi:10.1111/acer.12245.

Carmona-Perera, M., Reyes del Paso, G. A., Pérez-García, M. y Verdejo-García, A. (2013). Heart rate correlates of utilitarian moral decision-making in alcoholism. Drug and Alcohol Dependence, 133, 413-419. doi:10.1016/j.drugalcdep.2013.06.023.

Carou, M., Romero, E. y Luengo, M. A.(2017). Profiles of drug addicts in relation to personality variables and disorders. Adicciones, 29, 113-124. doi:10.20882/adicciones.889.

Chicharro, J., Pérez-García, A. M. y Sanjuán, P. (2011). Emotional responsiveness of substance abusers under outpatient treatment. Adicciones, 24, 59-68.

Cyders, M. A. y Smith, G. T. (2008). Emotion-based dispositions to rash action: positive and negative urgency. Psychological Bulletin, 134, 807-828. doi:10.1037/a0013341. 
Cyders, M. A., Zapolski, T. C .B., Combs, J. L., Settles, R. F., Fillmore, M. T. y Smith, G. T. (2010). Experimental effect of positive urgency on negative outcomes from risktaking and on increased alcohol consumption. Psychology of Addictive Behaviors, 24, 367-375.

Clark, D. B., Cornelius, J. R., Kirisci, L. y Tarter, R. E. (2005). Childhood risk categories for adolescent substance involvement: a general liability typology. Drug and Alcohol Dependence, 77, 13-21. doi:10.1016/j.drugalcdep.2004.06.008.

Danko, S. G., Gracheva, L. V., Boytsova, Y. A. y Solovjeva, M. L. (2011). Induction of emotional states by reading aloud texts with various emotional valences and changes in the EEG power in the beta and gamma frequency bands. Human Physiology, 37, 555-558. doi:10.1134/ S0362119711040062.

Derogatis, L. R. (1977). SCL-90-R: Administration, scoring and procedures Manual I for the revised version of other instruments of the Psychopathology Rating Scale series. John Hopkins University, Baltimore.

Dom, G., Wilde, B., Hulstijn, W., Brink, W. y Sabbe, B. (2006). Decision-making deficits in alcohol-dependent patients with and without comorbid personality disorder. Alcohol Clinical and Experimental Research, 30, 16701677. doi:10.1111/j.1530-0277.2006.00202.x.

Dunn, B. D., Stefanovitch, I., Evans, D., Oliver, C., Hawkins, A. y Dalgleish, T. (2010). Can you feel the beat? Interoceptive awareness is an interactive function of anxiety-and depression-specific symptom dimensions. Behaviour Research and Therapy, 48, 1133-1138. doi:10.1016/j.brat.2010.07.006.

Fein, G., Klein, L. y Finn, P. (2004). Impairment on a simulated gambling task in long-term abstinent alcoholics. Alcohol Clinical and Experimental Research, 28, 1487-1491. doi:10.1097/01.ALC.0000141642.39065.9B.

Fernández-Serrano, M. J., Pérez-García, M., Schmidt Río-Valle, J. y Verdejo-García, A. (2010). Neuropsychological consequences of alcohol and drug abuse on different components of executive functions. Journal of Psychopharmacology, 24, 1317-1332. doi:10.1177/0269881109349841.

Ferguson, M. L. y Katkin, E. S. (1996). Visceral perception, anhedonia, and emotion. Biological Psychology, 42, 131145. doi:10.1016/0301-0511(95)05151-1

First, M. B., 2007. Structured Clinical Interview for DSM-IVTR Axis I Disorders: SCID-I. Biometrics Research Department, New York State Psychiatric Institutute, New York.

Fishbein, D., Hyde, C., Eldreth, D., London, E. D., Matochik, J., Ernst, M., ...Kimes, A. (2005). Cognitive performance and autonomic reactivity in abstinent drug abusers and nonusers. Experimental and Clinical Psychopharmacology, 13, 25-40. doi:10.1037/1064-1297.13.1.25.

Foisy, M.L., Kornreich, C., Fobe, A., D'Hondt, L., Pelc, I., Hanak, C., Verbanck, P. y Philippot, P. (2007). Impaired emotional facial expression recognition in alcohol dependence: do these deficits persist with midterm abstinence? Alcohol Clinical and Experimental Research, 31, 404410. doi:10.1111/j.1530-0277.2006.00321.x.

Folstein, M. F., Folstein, S. E. y McHugh, P. R. (1975). Mini-Mental State: a practical method for grading the cognitive state of patients for the clinician. Journal of Psychiatric Research, 12, 189-98.

Garfield, J. B., Allen, N. B., Cheetham, A., Simmons, J. G. y Lubman, D. I. (2015). Attention to pleasant stimuli in early adolescence predicts alcohol-related problems in mid-adolescence. Biological Psychology, 108, 43-50. doi:10.1016/j.biopsycho.2015.03.014.

Gerra, G., Baldaro, B., Zaimovic, A., Moi, G., Bussandri, M., Raggi, M. A. y Bambrilla, F. (2003). Neuroendocrine responses to experimentally induced emotions among abstinent opioid-dependent subjects. Drug and Alcohol Dependence, 71, 25-35. doi:10.1016/S03768716(03)00065-6.

Goldstein, R. Z. y Volkow, N. D. (2002). Drug addiction and its underlying neurobiological basis: neuroimaging evidence for the involvement of the frontal cortex. American Journal of Psychiatry, 159, 1642-1652. doi:10.1176/ appi.ajp.159.10.1642.

Handelsman, L., Stein, J. A., Bernstein, D. P., Oppenheim, S. E., Rosenblum, A. y Magura, S. (2000). A latent variable analysis of coexisting emotional deficits in substance abusers: alexithymia, hostility, and PTSD. Addictive Behaviors, 25, 423-428. doi:10.1016/S03064603(99) 00010-6.

Hess, T. M. y Kotter-Grühn, D. (2011). Social knowledge and goal- based influences on social information processing in adulthood. Psychology and Aging, 26, 792-802. doi: $10.1037 / \mathrm{a} 0023775$.

Hudson, A., Jacques, S. y Stewart, S. H. (2013). Selective attention to emotional pictures as a function of gambling motives in problem and non-problem gamblers. Psychology of Addictive Behaviors, 27, 1079-1091. doi: 10.1037/ a0031863.

Kaiser, A., Bonsu, J. A., Charnigo, R. J., Milich, R. y Lynam, D. R. (2016). Impulsive Personality and Alcohol Use: Bidirectional Relations over One Year. Journal of Studies on Alcohol and Drugs, 77, 473-482. doi:10.15288/ jsad.2016.77.473.

Körner, N., Schmidt, P. y Soyka, M. (2015). Decision making and impulsiveness in abstinent alcohol-dependent people and healthy individuals: a neuropsychological examination. Substance Abuse Treatment, Prevention, and Policy, 10, 24. doi: 10.1186/s13011-015-0020-7.

Kornreich, C., Philippot, P., Foisy, M. L., Blairy, S., Raynaud, E., Dan, ...Verbanck, P. (2002). Impaired emotional facial expression recognition is associated with interpersonal problems in alcoholism. Alcohol and Alcoholism, 37, 394-400. 
Kreusch, F., Vilenne, A. y Quertemont, E. (2013). Response inhibition toward alcohol-related cues using an alcohol go/no-go task in problem and non-problem drinkers. Addictive Behaviors, 38, 2520-2528. doi:10.1016/j.addbeh.2013.04.007.

Lacey, J. I. y Lacey, B. C. (1970). Some autonomic-central nervous system interrelationships. In: Black, P. (Ed.), Physiological Correlates of Emotion. Academic Press, New York, pp. 205-227.

Lambe, L., Hudson, A. y Stewart, S. H. (2015). Drinking motives and attentional bias to affective stimuli in problem and non-problem drinkers. Psychology of Addictive Behaviors, 29, 312-316. doi:10.1037/adb0000021.

Lang, P. J., Öhman, A. y Vaitl, D. (1988). The International Affective Picture System [Photographic Slides]. Gainesville, FL: University of Florida, Center for Research in Psychophysiology.

Lang, P. J., Greenwald, M. K., Bradley, M. M. y Hamm, A. O. (1993). Looking at pictures: affective, facial, visceral, and behavioral reactions. Psychophysiology, 30, 261-273. doi:10.1111/j.1469-8986.1993.tb03352.x.

Lang, P. J. (1995). The emotion probe: Studies of motivation and attention. American Psychologist, 50, 372-385. doi:10.1037/0003-066X.50.5.372.

Lang, P. J. y Davis, M. (2006). Emotion, motivation, and the brain: Reflex foundations in animal and human research. Progress in Brain Research, 156, 3-29. doi:10.1016/ S0079-6123(06)56001-7.

Lole, L., Gonsalvez, C. J., Blaszczynski, A. y Clarke, A. R. (2012). Electrodermal activity reliably captures physiological differences between wins and losses during gambling on electronic machines. Psychophysiology, 49, 154163. doi:10.1111/j.1469-8986.2011.01290.x.

Lubman, D. I., Yücel, M., Kettle, J. W., Scaffidi, A., Mackenzie, T., Simmons, J. G. y Allen, N. B. (2009). Responsiveness to drug cues and natural rewards in opiate addiction: associations with later heroin use. Archives of General Psychiatry, 66, 205-212. doi:10.1001/archgenpsychiatry.2008.522.

Lubman, D. I., Peters, L. A., Mogg, K., Bradley, B. P. y Deakin, J. F. (2000). Attentional bias for drug cues in opiate dependence. Psychological Medicine, 30, 169-175. doi:10.1017/S0033291799001269.

Maurage, P., Campanella, S., Philippot, P., Martin, S. y Timary, P. (2008). Face processing in chronic alcoholism: a specific deficit for emotional features. Alcohol Clinical and Experimental Research, 32, 600-606. doi:10.1111/ j.1530-0277.2007.00611.x.

Mitchell, J. M., Fields, H. L., D’Esposito, M. y Boettiger, C. A. (2005). Impulsive responding in alcoholics. Alcohol Clinical and Experimental Research, 29, 2158-2169. doi:10.1097/01.alc.0000191755.63639.4a.

Millon, T. y Davis, R. D. (1997). The MCMI-III: present and future directions. Journal of Personality Assessment, 68, 6985. doi:10.1207/s15327752jpa6801_6.
Moeller, F. G., Barratt, E. S., Dougherty, D. M., Schmitz, J. M. y Swann, A. C. (2001). Psychiatric aspects of impulsivity. American Journal of Psychiatry, 2158, 1783-1793. doi:10.1176/appi.ajp.158.11.1783.

Moltó, J., Segarra, P., López, R., Esteller, A., Fonfría, A., Pastor, M. C. y Poy, R. (2013). Spanish adaptation of the "International Affective Picture System" (IAPS). Third part. Anals of Psicology, 29, 965-984.

Moselhy, H. F., Georgiou, G. y Kahn, A. (2001). Frontal lobe changes in alcoholism: A review of the literature. Alcohol and Alcoholism, 36, 357-368. doi:10.1093/alcalc/36.5.357.

Muller, S. E., Weijers, H. G., Boning, J. y Wiesbeck, G. A. (2008). Personality traits predict treatment outcome in alcohol-dependent patients. Neuropsychobiology, 57, 159164. doi:10.1159/000147469.

Najström, M. y Jansson, B. (2006). Unconscious responses to threatening pictures: interactive effect of trait anxiety and social desirability on skin conductance responses. Cognitive Behaviour Therapy, 35, 11-18. doi:10.1080/16506070510011566.

Naqvi, N. H. y Bechara, A. (2010). The insula and drug addiction: an interoceptive view of pleasure, urges, and decision-making. Brain Structure EF Function, 214, 435450. doi:10.1007/s00429-010-0268-7.

Nigg, J. T., Hinshaw, S. P. y Huang-Pollack, C. (2006). Disorders of attention and impulse regulation. In: Cicchetti, D., Cohen, D. (Ed.), Developmental psychopathology (pp. 358-403). New York: Wiley. doi:10.1002/9780470939406. ch9.

Ohannessian, C. M. y Hesselbrock, V. M. (2007). Do Personality Characteristics and Risk Taking Mediate the Relationship Between Paternal Substance Dependence and Adolescent Substance Use? Addictive Behaviors, 32, 1852-1862. doi:10.1016/j.addbeh.2006.12.017.

Palomba, D., Sarlo, M., Angrilli, A., Mini, A. y Stegagno, L. (2000). Cardiac responses associated with affective processing of unpleasant film stimuli. International Journal of Psychophysiology, 36, 45-57. doi:10.1016/S01678760(99)00099-9.

Park, S. Q., Kahnt, T., Beck, A., Cohen, M. X., Dolan, R. J., Wrase, J. y Heinz, A. (2010). Prefrontal cortex fails to learn from reward prediction errors in alcohol dependence. Journal of Neuroscience, 30, 7749-7753. doi:10.1523/JNEUROSCI.5587-09.2010.

Patton, J. H., Stanford, M. S. y Barratt, E. S. (1995). Factor structure of the Barratt impulsiveness scale. Journal of Clinical Psychology, 51, 768-774. doi.10.1002/10974679(199511)51:6\%3C768.

Paulus, M. P., Feinstein, J. S., Simmons, A. y Stein, M. B. (2004). Anterior cingulate activation in high trait anxious subjects is related to altered error processing during decision making. Biological Psychiatry, 55, 11791187. doi:10.1016/j.biopsych.2004.02.023. 
Petry, N. M. (2001). Delay discounting of money and alcohol in actively using alcoholics, currently abstinent alcoholics, and controls. Psychopharmacology, 154, 243-250. doi:10.1007/s002130000638.

Roedema, T. M. y Simons, R. F. (1999). Emotion processing deficit in alexithymia. Psychophysiology, 36, 379-387. doi:10.1017/S0048577299980290.

Robbins, S J.y Ehrman, R. N. (2004). The role of attentional bias in substance abuse. Behavioral and Cognitive Neuroscience Reviews, 3, 243-260. doi:10.1177/1534582305275423.

Robinson, T. E. y Berridge, K. C. (2001). Incentive-sensitization and addiction. Addiction, 96, 103-114.

Robinson, T. E y Berridge, K. C. (2003). Addiction. Annual Review of Psychology, 54, 25-53. doi:10.1046/j.13600443.2001.9611038.x.

Settles, R. E., Zapolski, T. C. B. y Smith, G. T. (2014). Longitudinal test of a developmental model of the transition to early drinking. Journal of Abnormal Psychology, 123, 141151. doi:10.1037/a0035670.

Schmidt, A. F., Eulenbruch, T., Langer, C. y Banger, M. (2013). Interoceptive Awareness, Tension Reduction Expectancies and Self-Reported Drinking Behavior. Alcohol and Alcoholism, 48, 472-477. doi:10.1093/alcalc/ agt024.

Smith, G. T. y Cyders, M. A. (2016). Integrating affect and impulsivity: The role of positive and negative urgency in substance use risk. Drug and Alcohol Dependence, 163, 3-12. doi:10.1016/j.drugalcdep.2015.08.038.

Sokolov, E. N. (1963). Higher Nervous Functions: The Orienting Reflex. Annual Review of Psychology, 25, 545580.

Stanford, M. S., Mathias, C. W., Dougherty, D. M., Lake, S. L., Anderson, N. E. y Patton, J. H. (2009). Fifty years of the Barratt Impulsiveness Scale: An update and review. Personality and Individual Differences, 47, 385-395. doi:10.1016/j.paid.2009.04.008.

Stephens, D. N. y Duka, T. (2008). Cognitive and emotional consequences of binge drinking: role of amygdala and prefrontal cortex. Philosophical Transactions of the Royal Society, 363, 3169-3179. doi:10.1098/rstb.2008.0097.

Tremayne, P. y Barry, R. J. (2001). Elite pistol shooters: physiological patterning of best vs. worst shots. International Journal of Psychophysiology, 41, 19-29. doi:10.1016/S01678760(00)00175-6.

Uekermann, J. y Daum, I. (2008). Social cognition in alcoholism: a link to prefrontal cortex dysfunction? Addiction, 103, 726-735. doi:10.1111/j.1360-0443.2008.02157.x.

Verdejo-García, A., López-Torrecillas, Aguilar de Arcos, F. y Pérez-García, M. (2005). Differential effects of MPMA, cocaine, and cannabis use severity on distinctive components of the executive functions in polysubstance users: a multiple regression analysis. Addictive Behaviors, 30, 89-101.

Verdejo-García, A., Bechara, A., Recknor, E. C. y Perez-Garcia, M. (2006). Executive dysfunction in substance de- pendent individuals during drug use and abstinence: an examination of the behavioral, cognitive, and emotional correlates of addiction. Journal of the International Neuropsychological Society, 12, 405-415.

Verdejo-García, A., Rivas-Pérez, C., Vilar-López, R. y Pérez-García, M. (2007). Strategic self-regulation, decision-making and emotion processing in poly-substance abusers in their first year of abstinence. Drug and Alcohol Dependence, 86, 139-146.

Verdejo-García, A., Lawrence, A. J. y Clark, L. (2008). Impulsivity as a vulnerability marker for substance-use disorders: review of findings from high-risk research, problem gamblers and genetic association studies. Neuroscience $\mathcal{E}$ Biobehavioral Reviews, 32, 777-810. doi:10.1016/j.drugalcdep.2006.05.024.

Verdejo-García, A. y Bechara, A. (2009). A somatic marker theory of addiction. Neuropharmacology, 56, 48-62. doi.10.1016/j.neuropharm.2008.07.035.

Verdejo-Garcia, A., Clark, L. y Dunn, B. D. (2012). The role of interoception in addiction: A critical review. Neuroscience E Biobehavioral Reviews, 36, 1857-1869. doi:10.1016/j.neubiorev.2012.05.007. 